\title{
Crossmodal Clustered Contrastive Learning: Grounding of Spoken Language to Gesture
}

\author{
Dong Won Lee \\ Carnegie Mellon University \\ Pittsburgh, PA, USA \\ dongwonl@andrew.cmu.edu
}

\author{
Chaitanya Ahuja \\ Carnegie Mellon University \\ Pittsburgh, USA \\ cahuja@andrew.cmu.edu
}

\author{
Louis-Philippe Morency \\ Carnegie Mellon University \\ Pittsburgh, PA, USA \\ morency@cs.cmu.edu
}

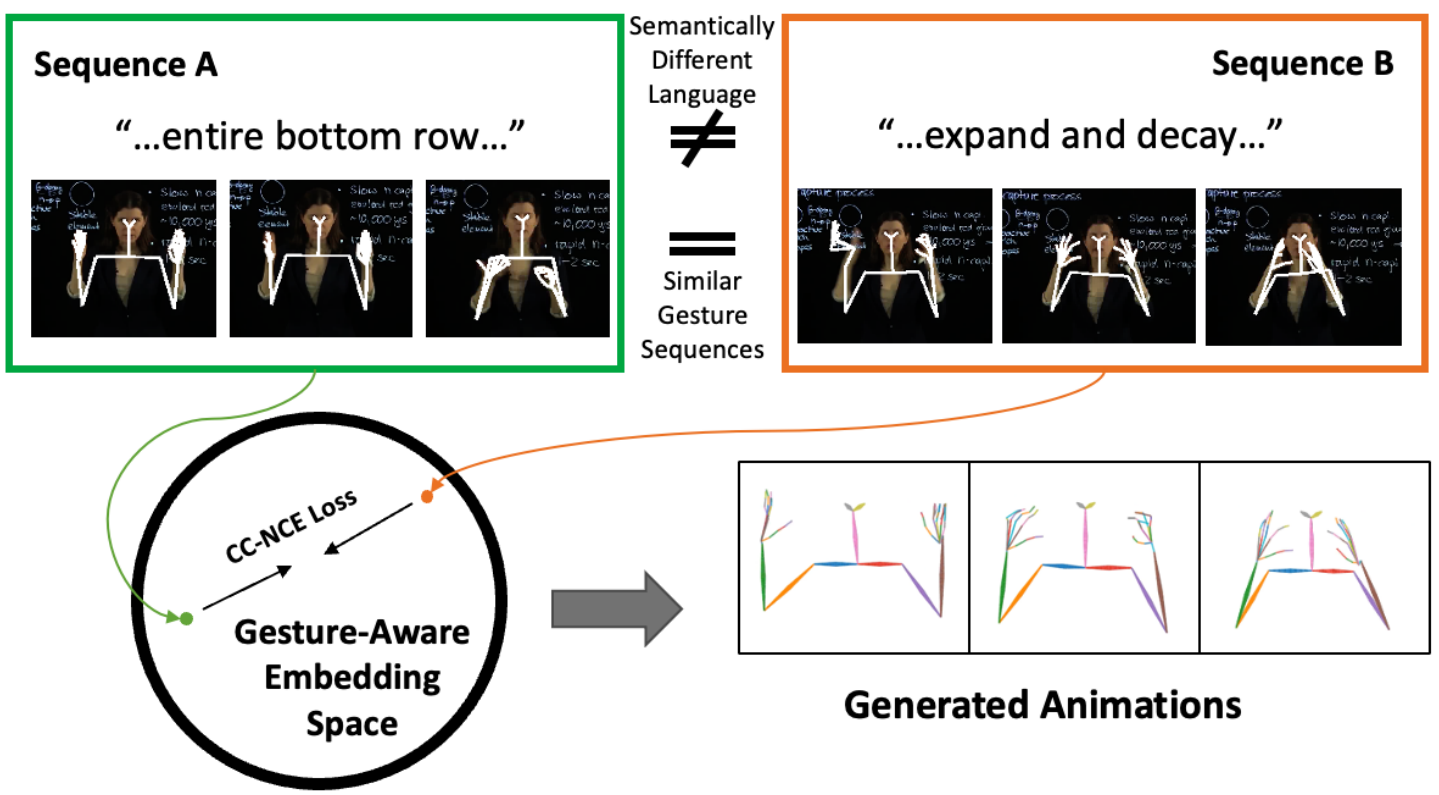

Figure 1: Consider two aligned sequences of spoken language phrases and gestures. The phrases, "entire bottom row" and "expand and decay" are semantically different, but accompanied by the same gesture. The language embeddings of these two sentences will be far apart in the semantic latent space. We want to guide the embeddings to be closer in the gesture-aware embedding space for gesture generation.

\begin{abstract}
Crossmodal grounding is a key technical challenge when generating relevant and well-timed gestures from spoken language. Often, the same gesture can accompany semantically different spoken language phrases which makes crossmodal grounding especially challenging. For example, a gesture (semi-circular with both hands) could co-occur with semantically different phrases "entire bottom row" (referring to a physical point) and "molecules expand and decay" (referring to a scientific phenomena). In this paper, we introduce a self-supervised approach to learn representations better suited to such many-to-one grounding relationships between spoken language and gestures. As part of this approach, we propose a
\end{abstract}

\section{(c) (i)}

This work is licensed under a Creative Commons Attribution International 4.0 License.

ICMI '21 Companion, October 18-22, 2021, Montréal, QC, Canada

(c) 2021 Copyright held by the owner/author(s).

ACM ISBN 978-1-4503-8471-1/21/10.

https://doi.org/10.1145/3461615.3485408 new contrastive loss function, Crossmodal Cluster NCE, that guides the model to learn spoken language representations which are consistent with the similarities in the gesture space. This gesture-aware space can help us generate more relevant gestures given language as input. We demonstrate the effectiveness of our approach on a publicly available dataset through quantitative and qualitative evaluations. Our proposed methodology significantly outperforms prior approaches for gestures-language grounding. Link to code: https://github.com/dondongwon/CC_NCE_GENEA.

\section{CCS CONCEPTS}

- Computing methodologies $\rightarrow$ Learning latent representations; Neural networks; Natural language processing.

\section{KEYWORDS}

Gesture generation; virtual agents; socially intelligent systems; co-speech gestures; multi-modal interaction; contrastive learning; crossmodal translation; deep learning 
ACM Reference Format:

Dong Won Lee, Chaitanya Ahuja, and Louis-Philippe Morency. 2021. Crossmodal Clustered Contrastive Learning: Grounding of Spoken Language to Gesture. In Companion Publication of the 2021 International Conference on Multimodal Interaction (ICMI '21 Companion), October 18-22, 2021, Montréal, QC, Canada. ACM, New York, NY, USA, 9 pages. https://doi.org/10.1145/ 3461615.3485408

\section{INTRODUCTION}

Nonverbal behaviours such as body posture, hand gestures and head nods play a crucial role in human communication [42]. Pointing at different objects, moving hands up-down in emphasis, and describing the outline of a shape are some of the many gestures that co-occur with verbal and vocal modalities of communication [21, 31]. When creating new robots or embodied virtual assistants designed to communicate with humans, it is important to generate gestures that are relevant with their expressed language and speech $[5,22,34]$.

Improving relevance of a gesture requires greater levels of grounding between spoken language and gestures, so that the generated gestures are more precise and related to the language. Improving the precision can be seen as restricting the mapping of semantically different language to a smaller subset of high quality gestures. Learning this mapping requires solving a many to-one relationship problem where the same gesture can be used for different spoken phrases [12,35] and modelling this relationship is a key technical challenge. For example, a gesture pointing at themselves is likely to co-occur with the spoken word 'me' as well as ' $m y$ heart'. This many-to-one relationship requires going beyond conventional losses that focus on solely relying on a reconstruction quantity to learn crossmodal grounding.

In this paper, we introduce the Crossmodal Cluster Noise Contrastive Estimation (CC-NCE) objective to learn a gesture-aware embedding space, where the similarities and dissimilarities in the gesture expressions are taken into account when learning language embeddings. Our objective guides the model to learn a gestureaware embedding space via a contrastive loss, where spoken language representations are consistent with the intra-cluster similarities and inter-cluster dissimilarities in the gesture space. In order to do so, we construct clusters in the gesture space in a self-supervised way. The constructed clusters is able to distinguish similar and dissimilar gestures, which can provide positive and negative samples for the contrastive loss. Therefore, given two different language sequences, CC-NCE guides their latent language representations to be close together if their accompanying gestures are in the same cluster and far apart if their gestures are in different clusters.

Our proposed CC-NCE Loss places an emphasis in learning the many-to-one grounding between language and gestures. CC-NCE is designed to provide additional incentive for the model to generate a smaller subset of higher quality gestures closer to the ground truth, with better performance on accuracy metrics. We conduct our experiments on the publicly available PATS dataset [2]. We study the effects of precision-coverage trade off, where the emphasis in precision and grounding can potentially come at a cost of decreased coverage.

\section{RELATED WORKS}

Language in Gesture Generation. A rule-based approach was proposed in an earlier study by Cassell et al. [6], where the behavior expression animation toolkit (BEAT) was developed to schedule behaviors, such as hand gestures, head nods and gaze. This approach was extended to utilize linguistic information from input text for selecting rules. [24, 27, 28, 30, 43].

Rule based approaches were replaced by deep conditional neural fields [8, 9] and Hidden Markov Models for prosody-driven head motion generation [38] and body motion generation [25, 26]. These use a dictionary of predefined animations, limiting the diversity of generated gestures. Soon, neural network based models were introduced, using unimodal inputs, specifically speech, to generate a sequence of gestures [18], head motions [37] and body motions $[2,3,13,14,40]$. On the other hand, Yoon et al. [45] uses only a text input for gesture generation. More recently, multimodal models utilizing both speech and language were developed. Kucherenko et al. [23] combines the two representations via early fusion. In order to account for the bi-modal relationship between language and audio in the input modalities, Ahuja et al. [1] utilizes a crossmodal attention mechanism to account for correlations between speech and language. It is important to note that many prior approaches $[1,2,14,23,44]$ rely on reconstruction losses (L1 distance between generated pose and ground truth) to learn the grounding between gestures and language. In this paper, we argue that the inclusion of an additional contrastive grounding loss is valuable to the model, specifically to learn the many-to-one mapping between spoken language and gestures.

Contrastive Learning. Contrastive learning has gained traction recently due to its success in self-supervised learning. Oord et al. [32] initially proposed the Contrastive Predictive Coding method to learn informative representations in a self-supervised manner via Noise Contrastive Estimation (NCE). NCE primarily relies on learning an parameterized encoder to estimate the true distribution (positives) against random noise (negatives). He et al. [19] proposed MoCo, which stores a long queue of samples, to insert as negatives to contrast with augmented anchor samples. Chen et al. [7] proposed SimCLR, which utilized large batch sizes, and eliminating the need for large stored dictionaries. Park et al. [33] offered a methodology called Patch-wise contrastive Loss, which maximizes the mutual information between corresponding input and output patches. More recently, a vein integrating clustering mechanisms with contrastive learning has been proposed, where unsupervised clusters are built in a unimodal space and noise contrastive estimation is applied $[4,20,29,39]$. Finally, pertinent to our crossmodal task, Udandarao et al. [41] projects each modality into a joint embedding space where both modalities are present. Then, they used supervised labels to retain intra-class and inter-class relationships for clusters in the joint space. Furthermore, their methods are designed for downstream discriminative tasks, whereas our task is generative. A key distinction is that our work utilizes selfsupervision to construct clusters, specifically in the output modality. We utilize the clusters in the output modality such that the same nature in is preserved in the representations of the input modality. 


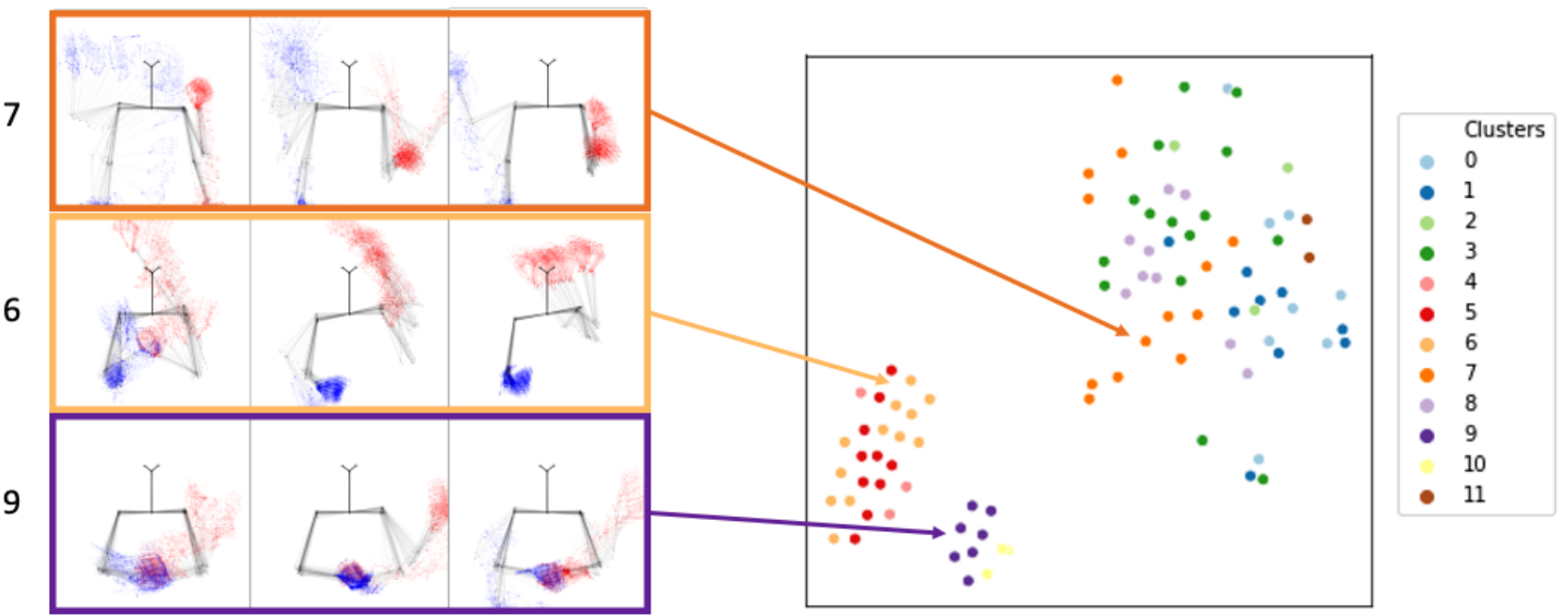

Figure 2: The heatmap on the left is an actual overlay of gesture sequences that were clustered together using our selfsupervised clustering algorithm, which demonstrates intracluster similarity and intracluster dissimilarity of gestures. The right is the actual t-SNE plot of language-embeddings after our CC-NCE Loss was applied. Our proposed approach brings spoken language embeddings closer to each other for similar gestures and faraway for dissimilar gestures.
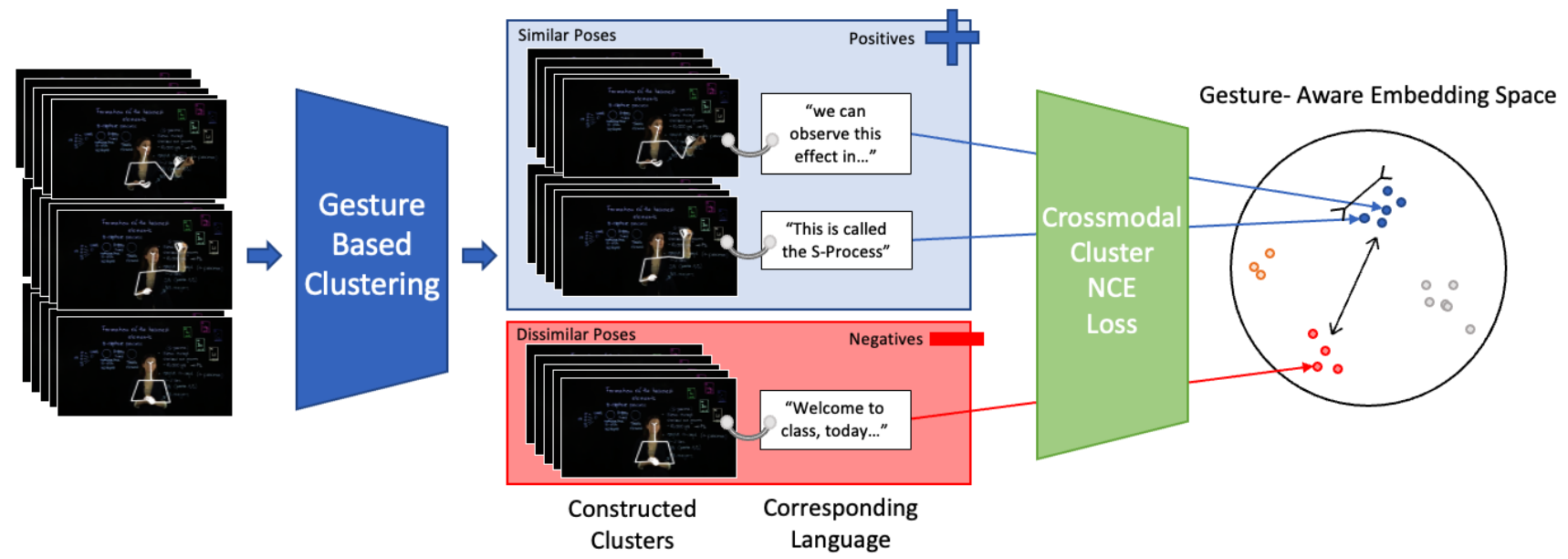

Figure 3: Our proposed approach of self-supervised clustering in the output space of gestures. The constructed clusters are used to find negative and positive samples for the Crossmodal Cluster NCE loss to learn a gesture-aware language embedding space.

\section{GESTURE GENERATION PROBLEM}

Our primary task is to learn a generative model which translates language (BERT tokens [11]) and speech (log-mel spectrograms) modalities to relevant co-speech gestures. To that end, we learn a joint embedding space where sentences $\mathrm{X}^{w}$ and speech signals $\mathrm{X}^{a}$ are mapped to latent embeddings $\mathbf{Z} \in \mathcal{Z}$ using an encoder $G_{e}$. These latent embeddings are further mapped to the space of human upper-body poses represented in temporal skeletal keypoints, (i.e
$\hat{\mathrm{Y}}^{p}$ ) using a decoder $G_{d}$ to optimize for the downstream task of gesture generation.

Formally, we are given a sentence of $K$ language tokens $\mathrm{X}^{w}=$ $\left[x_{1}^{w}, x_{2}^{w}, \ldots x_{K}^{w}\right]$ and a sequence of co-occurring speech features, $\mathrm{X}^{a}=\left[x_{1}^{a}, x_{2}^{a}, \ldots x_{T}^{a}\right]$. We want to predict a sequence of $\mathrm{T}$ gesture poses $\mathrm{Y}^{p}=\left[y_{1}^{p}, y_{2}^{p}, \ldots y_{T}^{p}\right]$ with $\mathrm{X}^{a}$ and $\mathrm{X}^{w}$ as input. Here $y_{t}^{p} \in$ $\mathcal{R}^{J \times 2}$ are the xy-coordinates for $t^{t h}$ frame for $J$ joints of the body 
skeleton. $x_{t}^{w} \in \mathcal{R}^{\mathcal{K}}$ is the $t^{t h}$ word embedding with dimension $K$ and $x_{t}^{a} \in \mathcal{R}^{\mathcal{M}}$ is the $t^{t h}$ speech frame with dimension $M$.

Thus, we have,

$$
\begin{aligned}
\mathbf{Z} & =G_{e}\left(\mathbf{X}^{a}, \mathbf{X}^{w} ; \theta\right) \\
\hat{\mathbf{Y}}^{p} & =G_{d}(\mathbf{Z} ; \psi)
\end{aligned}
$$

Parameters of this encoder-decoder model, $\theta, \psi$, are optimized with true poses $\mathrm{Y}^{p}$ as a training signal, which can be written as a reconstruction loss, $L_{r e c}(\theta)$ where we use the following L1 distance based on prior works $[1,2,14,23,44,45]$,

$$
\mathcal{L}_{r e c}(\theta, \psi)=\mathbb{E}_{\left.\mathbf{Y}^{p}, \mathbf{X}^{a}, \mathbf{X}^{w} \| \mathbf{Y}^{p}-G_{d}\left(G_{e}\left(\mathbf{X}^{a}, \mathbf{X}^{w}\right)\right)\right) \|_{1} .} .
$$

Often, as in GAN-based models [1, 14], adversarial losses [15] are included to alleviate the challenge of overly smooth generation and regression to the mean caused by reconstruction loss [14]. This adversarial loss is written as:

$$
\begin{aligned}
\mathcal{L}_{a d v}(\theta, \psi, \eta)= & \mathbb{E}_{\mathrm{Y}^{p}} \log D_{\eta}\left(\mathrm{Y}^{p}\right) \\
& +\mathbb{E}_{\mathbf{X}^{a}, \mathbf{X}^{w}} \log \left(1-D_{\eta}\left(G_{d}\left(G_{e}\left(\mathbf{X}^{a}, \mathbf{X}^{w}\right)\right)\right)\right.
\end{aligned}
$$
$\mathcal{L}$

The model is jointly trained to optimize the overall loss function

$$
\max _{\eta} \min _{\theta, \psi} \mathcal{L}_{r e c}(\theta, \psi)+\lambda \mathcal{L}_{a d v}(\theta, \psi, \eta)
$$

The above formulation is similar to previous works in gesture generation $[1,23,45]$.

\section{METHOD}

Our key contribution in this paper is to explicitly model the manyto-one mapping between spoken language and gestures in the latent space. This approach involves a two-step process, as shown in Figure 3. Our novel loss function $L_{c c-n c e}$ guides the aligned language representations to be close to each other if their corresponding ground truth gestures are in the same cluster, and far apart if their corresponding ground gestures gestures are not in the same cluster. Thereby, creating a gesture-aware embedding space. We also propose a clustering algorithm to find similar gestures in a self-supervised, online manner. The algorithm first approximates the distribution of pairwise gesture similarity scores. With the estimated mean and variance of the similarity score distribution, it constructs batch-wise clusters, which compares itself with the global clusters and then decides whether the batch-level cluster should be merged or form its own cluster.

Finally, the optimization of the combined objective function describes the full model,

$$
\max _{\eta} \min _{\theta, \psi} \mathcal{L}_{r e c}(\theta, \psi)+\mathcal{L}_{a d v}(\theta, \psi, \eta)+\mathcal{L}_{c c-n c e}(\theta, \psi)
$$

\subsection{Crossmodal Cluster NCE}

Given the same gesture, many different spoken phrases could accompany it, as shown in Figure 1. Therefore, even semantically different language embeddings corresponding to similar gesture sequences should be mapped closer together in the latent space.
In order to do so, we propose the Crossmodal Cluster Noise Contrastive Estimation Loss, inspired by the InfoNCE Loss [16, 17, 32] to learn the gesture-aware embedding space.

4.1.1 Gesture-aware Embedding Space. The InfoNCE Loss [16, 17, 32] first samples an anchor input sequence. Its augmentations are considered as positive samples, whereas the remaining elements within the batch (or a stored queue) are considered as negative samples $[7,19]$. We want to guide the language latent space to be close together for similar gesture sequences and far apart from other dissimilar ones. Hence, sampling a positive or negative sample from the dataset requires additional knowledge of the output gesture modality. To tackle this challenge, we construct unsupervised clusters in the output gesture modality, which is described in the next section, 4.1.2.

With these constructed clusters in the output gesture-domain, we want to coerce the corresponding language embeddings to mimic the inter-cluster and intra-cluster relationships in the gesture space. We are given an anchor sequence with ground truth gesture sequences and the corresponding language embeddings, $[y, z]$ respectively. We are also given global clusters of gesture sequences and their corresponding language embeddings. At this step, we want to find the cluster which contains gesture sequences that are most similar to the anchor gesture sequence. Mathematically, given a set of clusters $C$, we find the most similar gesture sequence and the aligned language embeddings: $y_{c}^{+}, z_{c}^{+}=\operatorname{argmax}\left(\operatorname{Sim}\left(y_{c}, y\right)\right)$, $\left.\forall\left[y_{c}, z_{c}\right] \in C\right)$. Here, we used the dot product as the similarity metric. Given the anchor, $z$, we use the corresponding language embeddings of the most gesture-wise similar cluster as the positive samples $z_{c}^{+}$. The language embedding sequences in other clusters will be considered as negative samples $z_{c}^{-}=\left[C \backslash z_{c}^{+}\right]$. With this assignment, we utilize properly assigned samples, in our Crossmodal Cluster NCE .

$$
L_{c c-n c e}=-\mathbb{E}_{z}\left[\log \frac{\exp \left(F(z)^{T} F\left(z_{c}^{+}\right)\right)}{\exp \left(F(z)^{T} F\left(z_{c}^{+}\right)\right)+\exp \left(F(z)^{T} F\left(z_{c}^{-}\right)\right)}\right]
$$

The numerator encourages the semantically different language representations to be closer since they belong in the same gesture cluster. Given an anchor sequence $z$, and gesture-wise similar positive language embeddings $z_{c}^{+}$and their dissimilar negatives $z_{c}^{-}$, we feed these language embeddings into an encoder, which we denote as $F($.$) to learn the relationships in the gesture space. By doing so,$ the Crossmodal Cluster NCE as shown in Equation 7 guides the language embedding space to learn the similarities in the output domain and projects them into a gesture-aware embedding space.

4.1.2 Gesture-Based Clustering. We want to embed the knowledge of the many-to-one relationship between spoken language and gestures as shown in Figure 1. To do so, we need to find clusters of similar gestures to provide positive and negative samples for manyto-one grounding. Since we are not provided with annotations of similar gesture clusters, we must do this in a self-supervised way.

The construction of unsupervised clusters can be computationally heavy for large datasets and requires the number of clusters which comes at a cost of an additional hyperparameter. To combat these technical challenges, we propose an online approach for 


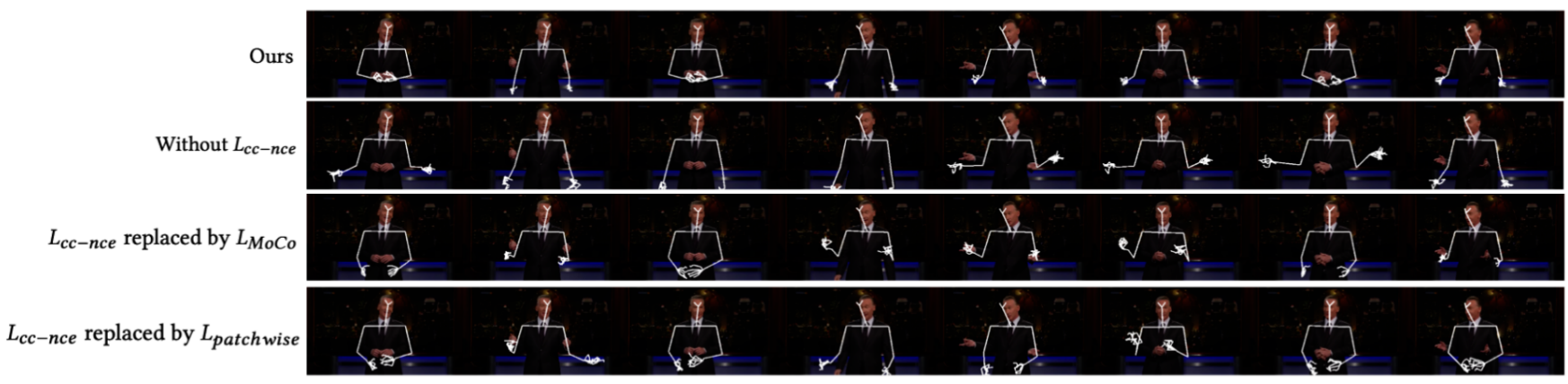

Figure 4: Generated keypoints superimposed on ground truth images for easy comparison. The usage of contrastive learning produces gestures closer to the ground truth

constructing these clusters where the number of clusters are dynamically chosen while learning the crossmodal translation model.

We iterate through the data and find the mean $\hat{\mu}$ and standard deviation $\hat{\sigma}$ of the pairwise dot-product similarity (referred to as $\mathrm{Sim}$ ) of two arbitrary sequences of gestures. This metric is updated using a moving average continuously. These metrics are utilized to find a threshold, which is used to determine whether two sequences are similar or not. For example, a sequence $x$ is considered to be similar to $y$, if $\operatorname{Sim}(x, y) \geq \hat{\mu}+\hat{\sigma}$. In essence, we are approximating the mean and variance of a Gumbell distribution, where the statistic of interest is $\operatorname{Sim}(x, y)$.

In practice, constructing and utilizing gesture-based clusters in an online manner is a two step process, (1) Batch Clustering and (2) Global Clustering, which is discussed below.

(1) Batch Clustering: The construction of the batch-wise cluster is important, as we can only compute the gradients with respect to the batch-wise embeddings and it would be infeasible to work with the global clusters due to computational limitations.

We describe the algorithm that is used to find the batch-level gesture clusters. In the first step, we calculate similarity metrics for an arbitrarily chosen anchor pose sequence, $y_{a}^{b}$, with the other pose sequences in the batch, $y^{b}[\sim L]$, where " $\sim L^{\prime \prime}$ are indices of sequences in the batch which has not been assigned to a cluster yet. The anchor sequence and sequences in the batch, which yield a similarity score greater than the threshold $\left(\operatorname{Sim}\left(y_{a}^{b}, y^{b}[\sim L]\right) \geq \hat{\mu}\right.$ $+\hat{\sigma})$, are assigned to a batch-wise cluster. Within the batch-level clustering, we want to discover clusters that are very different from each other. By assigning the next anchor sequence to the sequence with the lowest similarity score, the algorithm is able to find clusters that are very different from each other. An important advantage of this method is that it reduces the number of computations that needs to be computed. With this new anchor, the previously mentioned steps are applied recursively until all the sequences are assigned and we get a batch-wise dictionary of clusters, Batch ${ }_{D}$. Throughout this process, the latent embeddings corresponding to these gesturewise clusters are saved together. We refer the readers to Algorithm 1 in the appendix for more details.

(2) Global Clustering: After we obtain this batch-level dictionary of clusters, Batch ${ }_{D}$, we update the global dictionary of clusters Global $_{D}$. For each of the batch clusters, we sample a single sequence, $y_{\text {samp }}^{b}$, from the batch cluster $y^{b}$. Then, a sequence is sampled from each of the clusters in the global clusters $y^{g}$, we denote this as $y_{\text {samp }}^{g}$, which contains $\mid$ Global $_{D} \mid$ sequences. We check whether $y_{\text {samp }}^{b}$ sequence belongs in an existing cluster in Global $_{D}$ with the same thresholding logic: $\operatorname{Sim}\left(y_{\text {samp }}^{b}, y_{\text {samp }}^{g}\right) \geq \hat{\mu}+\hat{\sigma}$. If there exists a pair in that exceeds the threshold, we merge the batch cluster to the global cluster with the highest similarity value. Otherwise, we assign the batch cluster as a new global cluster in Global $_{D}$. Similarly to the batch clustering method, we save the corresponding latent embeddings in the global dictionary as well. We refer the readers Algorithm 2 for detailed description.

To tie this all back to our CC-NCE in Equation 7, we have $\left[y_{c b}^{b}, z_{c b}^{b}\right] \in$ Batch $_{D}$ and $\left[y_{c g}^{g}, z_{c g}^{g}\right] \in$ Global $_{D}$, where $c b$ indicates cluster index for the batch and $c g$ for the global dictionary. During training, given the i-th batch-level cluster, $\left[y_{i}^{b}, z_{i}^{b}\right]$, we treat the language embeddings, $z_{i}^{b}$, as the anchor sequences, because we want the language embeddings to learn the relationships present in the gesture space. Then, we find the most gesture-wise similar cluster in the global dictionary $y_{i}^{+}, z_{i}^{+}=\operatorname{argmax}\left(\operatorname{Sim}\left(y_{c g}^{g}, y_{i}^{b}\right)\right), \forall\left[y_{c g}^{g}, z_{c g}^{g}\right] \in$ Global $_{D}$ ). We use the corresponding language embeddings of the most similar global cluster as the positive samples $z_{i}^{+}$. The language embedding sequences in other clusters in the global dictionary will be considered as negative samples $z_{i}^{-}=\left[\right.$Global $\left._{D} \backslash z_{i}^{+}\right]$. With this assignment, we utilize properly assigned samples in our Crossmodal Cluster NCE in Equation 7.

\section{EXPERIMENTAL SETUP}

\subsection{Dataset}

We use the PATS dataset $[1,2,14]$ as the benchmark to measure performance. It consists of aligned body poses, audio, and transcripts for 25 speakers. We choose five speakers (maher, bee, lec_cosmic, oliver and colbert) with a wide range of linguistic content and contrasting gesture styles for our experiments.

\subsection{Baselines}

We utilize the Multimodal Multi-Scale Transformer based GANarchitecture [1] as a primary building block of our proposed model. To the best of our knowledge, there have been no previous approaches that explicitly learn gesture-guided semantic spaces with 


\begin{tabular}{|c|c|c|c|c|c|c|}
\hline Model & \multicolumn{6}{|c|}{$\mathrm{L} 1 \downarrow$} \\
\hline Speaker: & maher & bee & lec_cosmic & oliver & colbert & Mean \\
\hline Ours & $0.881 \pm 0.02$ & $0.918 \pm 0.017$ & $0.737 \pm 0.032$ & $0.777 \pm 0.02$ & $0.096 \pm 0.007$ & $0.682 \pm 0.007$ \\
\hline Without $L_{c c-n c e}[1]$ & $0.992 \pm 0.024$ & $0.955 \pm 0.036$ & $0.765 \pm 0.046$ & $\mathbf{0 . 7 7 5} \pm 0.025$ & $0.092 \pm 0.004$ & $0.716 \pm 0.006$ \\
\hline$L_{c c-n c e}$ replaced by $L_{M o C o}[19]$ & $0.983 \pm 0.028$ & $0.94 \pm 0.058$ & $0.763 \pm 0.042$ & $0.781 \pm 0.021$ & $0.091 \pm 0.002$ & $0.771 \pm 0.086$ \\
\hline$L_{c c-n c e}$ replaced by $L_{\text {patchwise }}[33]$ & $0.951 \pm 0.033$ & $0.937 \pm 0.019$ & $0.731 \pm 0.019$ & $0.874 \pm 0.124$ & $0.096 \pm 0.003$ & $0.769 \pm 0.085$ \\
\hline
\end{tabular}

Table 1: Ablation of various contrastive loss mechanisms for 5 speakers in PATS [2] for gesture generation in terms of precision (L1). Ours utilizes the proposed $L_{c c-n c e}$ loss. Without $L_{c c-n c e}$ utilizes no contrastive learning at all, as proposed in [1]. $L_{c c-n c e}$ is replaced by two other contrastive learning mechanisms $L_{M o C o}$ [19] and $L_{\text {patch }}$ wise [33] for comparison.

contrastive loss functions. We compare our model with other selfsupervised approaches, $L_{M o C o}$ and $L_{\text {patchwise }}$, by replacing the loss function $L_{c c-n c e}$ in Equation 6.

$L_{c c-n c e}$ replaced by $L_{M o C o}$ : The contrastive learning proposed in MoCo [19] builds a large queue of data samples. The queue is referenced to find positive samples, if the encoded views are from the same image. Otherwise, the remaining elements are considered to be negative. This model is similar to our proposed $L_{c c-n c e}$, without the utilization of clustering in the gesture space to assign positive and negative labels and relying on data augmentation and noise sampling for this assignment.

$L_{c c-n c e}$ replaced by $L_{\text {patchwise }}$ : Another contrastive learning approach: patch-wise contrastive learning [33] uses a specific contrastive loss, which maximizes the mutual information between the corresponding input and output patches. The mechanism aligns corresponding input-output patches at specific regions, which allows it discretize inputs into patches and use them as positives and negatives.

Without $L_{c c-n c e}$ : We also compare our proposed model without the $L_{c c-n c e}$ loss function which boils down to the backbone model [1].

\subsection{Experimental Methodology}

In order to measure the precision and grounding of the generations, specifically relevance and timing of the gestures, we report the L1 distance between generated and ground-truth gestures. To measure the distribution in the gesture domain, we utilize the Fréchet Inception Distance (FID), which has been used in comparing gesture distributions $[1,44]$, which measures the distance between the distributions of the output generated poses and the ground truth. These results are included in the Appendix Table 2,

\subsection{Implementation Details}

The baselines were all trained with their respective hyperparameters. We remove the AISLE adaptive reweighting mechanism in [1] for our backbone model as it feeds in various samples repeatedly into the model. Because our model constructs clusters in an online manner, the re-sampling method causes the clusters to be constructed with repeated samples, which can be problematic. Furthermore, in order to initialize the global mean and standard deviation of similarity scores for two pairwise sequences $\hat{\mu}$, $\hat{\sigma}$ for online self-supervised clustering, we iterate through the data for two epochs to find the mean $\hat{\mu}$ and standard deviation $\hat{\sigma}$ of the pairwise dot product similarity (referred to as Sim) of two arbitrary sequences of poses, similar to approximating the mean and variance of a Gum-bell distribution. During this time, the Crossmodal Cluster NCE is not applied. Finally, the encoder in 4.1 which learns our gesture-aware embedding space is based on a U-Net structure [36].

\section{RESULTS AND DISCUSSION}

We substantiate our results by testing on five sampled speakers from the PATS dataset, displayed in Table 1. We give detailed metrics for each speaker for the precision metric L1, FID and their respective means.

Impact on Precision: Our proposed model with the inclusion of CC-NCE produces better L1 scores than other baselines (Table 1). We see a significant decrease in L1 scores. This implies that our CC-NCE model produces better well-timed and relevant gestures compared to other baselines. Specifically, we see that other contrastive learning approaches, $L_{M o C o}$ and $L_{\text {patchwise }}$ have worse L1 scores than that of the baseline model without any contrastive learning (Without $L_{c c-n c e}$ ). This additionally shows that our proposed method of constructing clusters in the output domain and coercing the model to learn a gesture-aware embedding space is beneficial.

Impact on Coverage: Although our results show improvements in precision, there are important limitations to consider. We refer the readers to Table 2. We see our model having worse FID scores, which represents the coverage of the generated distribution. The no contrastive learning [1] method, which uses an adaptive importance sampling approach for better performance in coverage, produces the best results. We are providing additional incentive for the model to generate a limited subset of gestures, as we are mapping a large language space to a smaller subspace of gestures. Therefore, a decrease in the FID scores is explained by the trade-off between coverage and precision. The qualitative figure shown in Fig. 4 shed additional insight to this trade-off.

Impact of $L_{c c-n c e}$ : We demonstrate the effectiveness of our Crossmodal Cluster NCE Loss and display the resulting gesture-aware embedding space ( $t$-SNE plot) and self-supervised gesture clusters (heatmap) in Figure 2. Firstly, the heatmap plot demonstrate that the self-supervised clustered pose sequences are indeed similar. Each row of the heatmap displays an overlay of three individual 64 -frame sequences in a specific cluster (indices $6,7,9$ ). The red 


\begin{tabular}{|l|c|c|c|c|c|c|}
\hline Model & \multicolumn{5}{|c|}{ FID $\downarrow$} \\
\hline Speaker: & maher & bee & lec_cosmic & oliver & colbert & Mean \\
\hline Ours & $48.52 \pm 5.39$ & $100.03 \pm 20.74$ & $44.43 \pm 9.71$ & $54.06 \pm 9.38$ & $5.85 \pm 0.84$ & $50.58 \pm 7.15$ \\
\hline Without $L_{c c-n c e}[1]$ & $\mathbf{2 1 . 3 8} \pm \mathbf{3 . 8 9}$ & $\mathbf{6 5 . 6 7} \pm \mathbf{1 1 . 3 5}$ & $\mathbf{2 3 . 1 4} \pm \mathbf{1 1 . 0 3}$ & $\mathbf{4 6 . 4 8} \pm \mathbf{1 . 1 2}$ & $6.77 \pm 0.05$ & $\mathbf{3 2 . 6 9} \pm \mathbf{3 . 9 0}$ \\
\hline$L_{c c-n c e}$ replaced by $L_{\text {MoCo }}[19]$ & $32.15 \pm 20.83$ & $74.892 \pm 24.17$ & $27.38 \pm 16.71$ & $48.78 \pm 2.13$ & $6.57 \pm 0.16$ & $39.66 \pm 12.38$ \\
\hline$L_{c c-n c e}$ replaced by $L_{\text {patchwise }}[33]$ & $26.45 \pm 3.74$ & $70.23 \pm 10.52$ & $38.95 \pm 4.02$ & $49.47 \pm 9.47$ & $\mathbf{5 . 4 8} \pm \mathbf{0 . 8 5}$ & $33.30 \pm 3.74$ \\
\hline
\end{tabular}

Table 2: Ablation of various contrastive loss mechanisms for 5 speakers in PATS [2] for gesture generation in terms of coverage (FID). Ours utilizes the proposed $L_{c c-n c e}$ loss. Without $L_{c c-n c e}$ utilizes no contrastive learning at all, as proposed in [1]. $L_{c c-n c e}$ is replaced by two other contrastive learning mechanisms $L_{M o C o}$ [19] and $L_{\text {patchwise }}$ [33] for comparison.

color indicates movements in the right arm and the blue color represents that of the left arm. For cluster 7, the gesture is dominated by a raised right arm and an up and down motion of the left arm. For cluster 9, the speaker is at their rest pose, with slight up and down movements of the right arm. Finally, for cluster 6, we can see that the left arm is mainly static, with movements on the right arm. Visually, we can see that clusters 6 and 9 are quite similar, with movements mainly dominated by the right arm, whereas cluster 7 is quite different. In the gesture-aware embedding space, we also see that clusters 6 and 9 lie in closer regions in the t-SNE plot of the language representations, in comparison to that of cluster 7. This demonstrates that the intra-cluster and inter-cluster relationships for gesture similarity and dissimilarity is indeed preserved in the latent space as well. If the clustering information was not effectively transferred to the latent space, we would not be able to visually see the clusters in the t-SNE plot located in similar regions.

Qualitative Comparison: We refer the readers to Figure 4, which shows a rendering of each model's generated gestures superimposed on the ground truth images for easy comparison of the quality of the generations. Our generated gestures are close to the ground truth. Specifically, the many-to-one grounding between a smaller subset of gestures and language allows for less noisy generations, which are confined to a smaller higher quality subset of gestures, which is due to the clustered gesture-aware embedding space. The baseline model, denoted as "Without $L_{c c-n c e}$ " [1], whose model architecture is designed around minimizing the distribution difference between the generation and the ground truth, produces gestures that are quite diverse but nonetheless divulges from the ground truth. On the other hand, the contrastive learning based methods Ours, $L_{M o C o}[19]$, and $L_{\text {patchwise }}$ [33], seem to generate more relevant and precision gestures, which shows higher levels of grounding.

Limitations and Future Work Certain speakers with greater diversity contain gesture sequences that are quite different from that of the majority of the cluster. The key challenge lies in constructing self-supervised clusters in both the temporal and spatial dimension. On the other hand, converting this into a supervised task, with annotations collected for gesture clusters, would make CC-NCE even more effective. Secondly, we observe that the generated gestures have finger keypoints that are abnormal for specific speakers. This may be due to the fact that the CC-NCE is confounding the final objective function with the reconstruction loss, causing the output generations to be noisy (especially since finger keypoints in the data are noisy due to its versatile movements). Finally, excessive grounding information may contribute to mode collapse, as it encourages the model to produce similar subset of gestures. Studies need to be done to encourage grounding while preventing convergence to a smaller subset of modes.

This approach shows promise in a wide variety of crossmodal tasks to enforce stronger levels of grounding in a self-supervised manner. Furthermore, pertinent to our task of gesture generation, a more fine-grained clustering could be done spatially (clustering based on left arm/right arm movements separately) and temporally (considering differing levels of granularity). Finally, exploring other options of domain-specific similarity metrics, such as DTW [10] for speed-invariant gestures, may help construct better clusters.

\section{CONCLUSION}

In this paper, we studied crossmodal grounding in the context of many-to-one mapping between spoken language and gestures for the task of co-speech gesture generation. We introduced a new contrastive loss function Crossmodal Cluster NCE loss, which guides the latent space to learn the similarities and dissimilarities in the constructed clusters in the gesture domain. Furthermore, we offered a mechanism to cluster temporal sequences in a self-supervised and online fashion. We demonstrated the effectiveness of this approach on a publicly available dataset, which indicated that our proposed methodology outperformed prior approaches in grounding gestures to language. We also observe, in-line with the precision-coverage trade-off, that encouraging higher precision could potentially degrade the coverage of the generated gestures.

\section{REFERENCES}

[1] Chaitanya Ahuja, Dong Won Lee, Ryo Ishii, and Louis-Philippe Morency. 2020. No Gestures Left Behind: Learning Relationships between Spoken Language and Freeform Gestures. In Proceedings of the 2020 Conference on Empirical Methods in Natural Language Processing: Findings. 1884-1895.

[2] Chaitanya Ahuja, Dong Won Lee, Yukiko I Nakano, and Louis-Philippe Morency. 2020. Style Transfer for Co-Speech Gesture Animation: A Multi-Speaker Conditional-Mixture Approach. Proceedings of the European Conference on Computer Vision (2020).

[3] Chaitanya Ahuja, Shugao Ma, Louis-Philippe Morency, and Yaser Sheikh. 2019. To React or not to React: End-to-End Visual Pose Forecasting for Personalized Avatar during Dyadic Conversations. In 2019 International Conference on Multimodal Interaction. ACM, 74-84.

[4] Alexei Baevski, Henry Zhou, Abdelrahman Mohamed, and Michael Auli. 2020. wav2vec 2.0: A framework for self-supervised learning of speech representations. arXiv preprint arXiv:2006.11477 (2020)

[5] Jeremy N Bailenson, Nick Yee, Dan Merget, and Ralph Schroeder. 2006. The effect of behavioral realism and form realism of real-time avatar faces on verbal disclosure, nonverbal disclosure, emotion recognition, and copresence in dyadic 
interaction. Presence: Teleoperators and Virtual Environments 15, 4 (2006), 359-372.

[6] Justine Cassell, Hannes Högni Vilhjálmsson, and Timothy Bickmore. 2001. BEAT: the Behavior Expression Animation Toolkit. In the 28th annual conference on Computer graphics and interactive techniques (SIGGRAPH '01). 477-486. https: //doi.org/10.1145/383259.383315

[7] Ting Chen, Simon Kornblith, Mohammad Norouzi, and Geoffrey Hinton. 2020 A simple framework for contrastive learning of visual representations. arXiv preprint arXiv:2002.05709 (2020).

[8] Chung-Cheng Chiu and Stacy Marsella. 2014. Gesture generation with lowdimensional embeddings. In Proceedings of the 2014 international conference on Autonomous agents and multi-agent systems. 781-788.

[9] Chung Cheng Chiu, Louis Philippe Morency, and Stacy Marsella. 2015. Predicting co-verbal gestures: A deep and temporal modeling approach. In Proceedings of the 15th international conference on Intelligent virtual agents (IVA2015), Vol. 9238. 152-166. https://doi.org/10.1007/978-3-319-21996-7_17

[10] Marco Cuturi and Mathieu Blondel. 2017. Soft-DTW: a differentiable loss function for time-series. arXiv preprint arXiv:1703.01541 (2017).

[11] Jacob Devlin, Ming-Wei Chang, Kenton Lee, and Kristina Toutanova. 2018. Bert: Pre-training of deep bidirectional transformers for language understanding. arXiv preprint arXiv:1810.04805 (2018).

[12] Shichang Feng, Zhiquan Feng, and Liujuan Cao. 2019. Many-to-One Gesture-toCommand Flexible Mapping Approach for Smart Teaching Interface Interaction. IEEE Access 7 (2019), 179517-179531.

[13] Ylva Ferstl, Michael Neff, and Rachel McDonnell. 2019. Multi-objective adversarial gesture generation. (2019)

[14] Shiry Ginosar, Amir Bar, Gefen Kohavi, Caroline Chan, Andrew Owens, and Jitendra Malik. 2019. Learning Individual Styles of Conversational Gesture. In Proceedings of the IEEE Conference on Computer Vision and Pattern Recognition. 3497-3506.

[15] Ian Goodfellow, Jean Pouget-Abadie, Mehdi Mirza, Bing Xu, David Warde-Farley, Sherjil Ozair, Aaron Courville, and Yoshua Bengio. 2014. Generative adversarial nets. In Advances in neural information processing systems. 2672-2680.

[16] Michael Gutmann and Aapo Hyvärinen. 2010. Noise-contrastive estimation: A new estimation principle for unnormalized statistical models. In Proceedings of the Thirteenth International Conference on Artificial Intelligence and Statistics. JMLR Workshop and Conference Proceedings, 297-304.

[17] Michael U Gutmann and Aapo Hyvärinen. 2012. Noise-Contrastive Estimation of Unnormalized Statistical Models, with Applications to Natural Image Statistics. fournal of Machine Learning Research 13, 2 (2012).

[18] Dai Hasegawa, Naoshi Kaneko, Shinichi Shirakawa, Hiroshi Sakuta, and Kazuhiko Sumi. 2018. Evaluation of Speech-to-Gesture Generation Using Bi-Directional LSTM Network. In Proceedings of the 18th International Conference on Intelligent Virtual Agents (IVA18). 79-86.

[19] Kaiming He, Haoqi Fan, Yuxin Wu, Saining Xie, and Ross Girshick. 2020. Momentum contrast for unsupervised visual representation learning. In Proceedings of the IEEE/CVF Conference on Computer Vision and Pattern Recognition. 9729-9738.

[20] Wei-Ning Hsu, Yao-Hung Hubert Tsai, Benjamin Bolte, Ruslan Salakhutdinov, and Abdelrahman Mohamed. 2021. HuBERT: How much can a bad teacher benefit ASR pre-training?. In ICASSP 2021-2021 IEEE International Conference on Acoustics, Speech and Signal Processing (ICASSP). IEEE, 6533-6537.

[21] Adam Kendon. 1980. Gesture and speech: two aspects of the process of utterance. In Nonverbal Communication and Language, M. R. Key (Ed.). 207-227.

[22] Taras Kucherenko, Dai Hasegawa, Gustav Eje Henter, Naoshi Kaneko, and Hedvig Kjellström. 2019. Analyzing Input and Output Representations for Speech-Driven Gesture Generation. arXiv preprint arXiv:1903.03369 (2019).

[23] Taras Kucherenko, Patrik Jonell, Sanne van Waveren, Gustav Eje Henter, Simon Alexanderson, Iolanda Leite, and Hedvig Kjellström. 2020. Gesticulator: A framework for semantically-aware speech-driven gesture generation. arXiv preprint arXiv:2001.09326 (2020).

[24] Jina Lee and Stacy Marsella. 2006. Nonverbal behavior generator for embodied conversational agents. In Proceedings of the 6th international conference on Intelligent virtual agents (IVA2006). 243-255.

[25] Sergey Levine, Philipp Krähenbühl, Sebastian Thrun, and Vladlen Koltun. 2010 Gesture Controllers. ACM Trans. Graph. 29, 4, Article 124 (July 2010), 11 pages.

[26] Sergey Levine, Christian Theobalt, and Vladlen Koltun. 2009. Real-time Prosodydriven Synthesis of Body Language. ACM Trans. Graph. 28, 5, Article 172 (Dec 2009), 10 pages.

[27] Margot Lhommet and Stacy Marsella. 2016. From embodied metaphors to metaphoric gestures. CogSci (2016), 788-793.

[28] Margot Lhommet, Yuyu Xu, and Stacy Marsella. 2015. Cerebella: Automatic Generation of Nonverbal Behavior for Virtual Humans. In Proceedings of the Twenty-Ninth AAAI Conference on Artificial Intelligence. 4303-4304.

[29] Junnan Li, Pan Zhou, Caiming Xiong, Richard Socher, and Steven CH Hoi. 2020 Prototypical contrastive learning of unsupervised representations. arXiv preprint arXiv:2005.04966 (2020).

[30] Stacy Marsella, Yuyu Xu, Margaux Lhommet, Andrew Feng, Stefan Scherer, and Ari Shapiro. 2013. Virtual character performance from speech. In Symposium on Computer Animation. 25-35. https://doi.org/10.1145/2485895.2485900
[31] David McNeill. 1992. Hand and mind: What gestures reveal about thought. University of Chicago Press.

[32] Aaron van den Oord, Yazhe Li, and Oriol Vinyals. 2018. Representation learning with contrastive predictive coding. arXiv preprint arXiv:1807.03748 (2018).

[33] Taesung Park, Alexei A Efros, Richard Zhang, and Jun-Yan Zhu. 2020. Contrastive learning for unpaired image-to-image translation. In European Conference on Computer Vision. Springer, 319-345.

[34] Catherine Pelachaud. 2009. Studies on gesture expressivity for a virtual agent. Speech Communication 51, 7 (2009), 630-639.

[35] Yosra Rekik, Laurent Grisoni, and Nicolas Roussel. 2013. Towards many gestures to one command: A user study for tabletops. In IFIP Conference on HumanComputer Interaction. Springer, 246-263.

[36] Olaf Ronneberger, Philipp Fischer, and Thomas Brox. 2015. U-net: Convolutiona networks for biomedical image segmentation. In International Conference on Medical image computing and computer-assisted intervention. Springer, 234-241.

[37] Najmeh Sadoughi and Carlos Busso. 2018. Novel Realizations of Speech-Driven Head Movements with Generative Adversarial Networks. 6169-6173. https: //doi.org/10.1109/ICASSP.2018.8461967

[38] Mehmet E. Sargin, Yucel Yemez, Engin Erzin, and Ahmet M. Tekalp. 2008. Analysis of head gesture and prosody patterns for prosody-driven head-gesture animation. IEEE Trans. Pattern Anal. Mach. Intell. 30 (2008), 1330-1345. https://doi.org/10. 1109/TPAMI.2007.70797

[39] Steffen Schneider, Alexei Baevski, Ronan Collobert, and Michael Auli. 2019. wav2vec: Unsupervised pre-training for speech recognition. arXiv preprint arXiv:1904.05862 (2019)

[40] Eli Shlizerman, Lucio Dery, Hayden Schoen, and Ira Kemelmacher. 2018. Audio to Body Dynamics. Proceedings / CVPR, IEEE Computer Society Conference on Computer Vision and Pattern Recognition. IEEE Computer Society Conference on Computer Vision and Pattern Recognition (06 2018).

[41] Vishaal Udandarao, Abhishek Maiti, Deepak Srivatsav, Suryatej Reddy Vyalla, Yifang Yin, and Rajiv Ratn Shah. 2020. Cobra: Contrastive bi-modal representation algorithm. arXiv preprint arXiv:2005.03687 (2020).

[42] Petra Wagner, Zofia Malisz, and Stefan Kopp. 2014. Gesture and speech in interaction: An overview.

[43] Yuyu Xu, Catherine Pelachaud, and Stacy Marsella. 2014. Compound gesture generation: A model based on ideational units. Lecture Notes in Computer Science (including subseries Lecture Notes in Artificial Intelligence and Lecture Notes in Bioinformatics) 8637 LNAI (2014), 477-491. https://doi.org/10.1007/978-3-31909767-1_58

[44] Youngwoo Yoon, Bok Cha, Joo-Haeng Lee, Minsu Jang, Jaeyeon Lee, Jaehong Kim, and Geehyuk Lee. 2020. Speech gesture generation from the trimodal context of text, audio, and speaker identity. ACM Transactions on Graphics (TOG) 39, 6 (2020), 1-16.

[45] Youngwoo Yoon, Woo-Ri Ko, Minsu Jang, Jaeyeon Lee, Jaehong Kim, and Geehyuk Lee. 2019. Robots learn social skills: End-to-end learning of co-speech gesture generation for humanoid robots. In 2019 International Conference on Robotics and Automation (ICRA). IEEE, 4303-4309. 


\section{A APPENDIX}

\section{A.1 Crossmodal Cluster NCE: Algorithmic details}

In Algorithms 1 and 2 we describe, in detail, Batch Clustering and Global Clustering that are key components for estimating our proposed CC-NCE model.

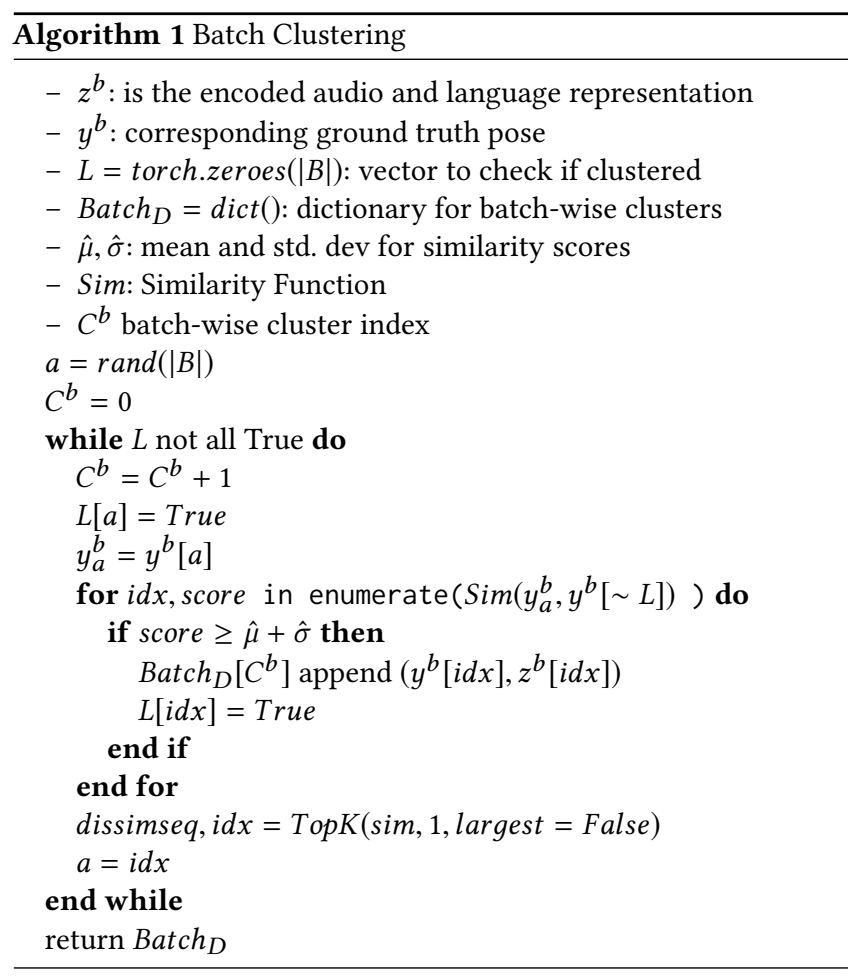

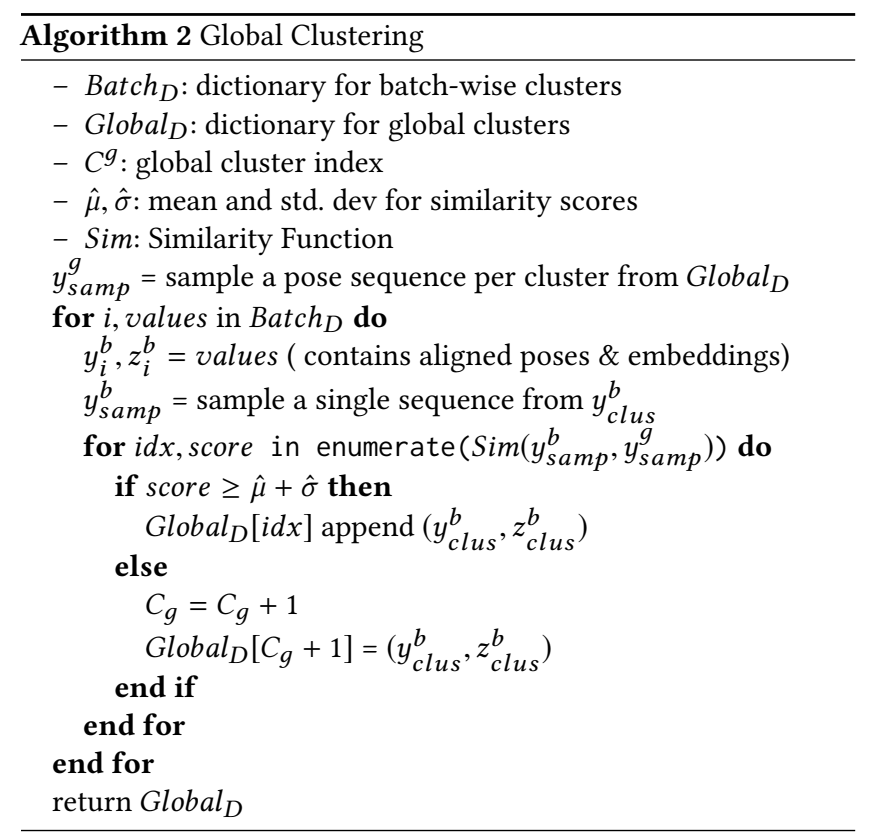

